\title{
Exclusive diffractive processes including saturation effects at next-to-leading order
}

\author{
Samuel Wallon* \\ Laboratoire de Physique Théorique (UMR 8627), CNRS, Univ. Paris-Sud, Université \\ Paris-Saclay, 91405, Orsay, France \& \\ Sorbonne Université, Faculté de Physique, 4 place Jussieu, 75252 Paris Cedex 05, France \\ E-mail: samuel.walloneth.u-psud.fr
}

\section{Renaud Boussarie}

Physics Department, Brookhaven National Laboratory, Upton, NY 11973, USA

Email: rboussarie@bnl.gov

\section{Andrey V. Grabovsky}

Budker Institute of Nuclear Physics, 11, Lavrenteva avenue, 630090, Novosibirsk, Russia \& Novosibirsk State University, 630090, 2, Pirogova street, Novosibirsk, Russia

Email: A.V.Grabovsky@inp.nsk.su

\section{Lech Szymanowski}

National Centre for Nuclear Research (NCBJ), 02-093 Warsaw, Poland

Email: Lech.Szymanowski@ncbj.gov.pl

In the framework of the QCD shock-wave approach, we review our results on the description of diffractive production of various final states (jets, meson) at next-to-leading order. This is applied to exclusive diffractive dijet electroproduction at HERA.

Light Cone 2019 - QCD on the light cone: from hadrons to heavy ions - LC2019

16-20 September 2019

Ecole Polytechnique, Palaiseau, France

*Speaker. 
The HERA research program revealed that almost $10 \%$ of the deep inelastic scattering (DIS) events were shown to contain a rapidity gap in the detectors between the proton remnants and the hadrons coming from the fragmentation region of the initial virtual photon. Among these events, exclusive diffractive production of dijets is particularly promising in order to distinguish between a collinear QCD factorized description involving distributions of partons inside the exchanged Pomeron [1], and a high-energy description in which the Pomeron is directly coupled to the hard subprocess. We here briefly report on this second description, including gluonic saturation within the QCD shockwave approach, which we then apply to ZEUS data.

\section{The QCD shockwave approach}

In a balanced frame, e.g. center-of-mass frame (c.m.f), consider a projectile scattering a target respectively flying almost along light-cone directions $n_{1}$ and $n_{2}$, with

$$
n_{1}=\sqrt{\frac{1}{2}}\left(1,0_{\perp}, 1\right), \quad n_{2}=\sqrt{\frac{1}{2}}\left(1,0_{\perp},-1\right), \quad\left(n_{1} \cdot n_{2}\right)=1 .
$$

Introducing lightcone coordinates

$$
x=\left(x^{0}, x^{1}, x^{2}, x^{3}\right) \rightarrow\left(x^{+}, x^{-}, \vec{x}\right) \quad \text { with } \quad x^{+}=x_{-}=\left(x \cdot n_{2}\right), \quad x^{-}=x_{+}=\left(x \cdot n_{1}\right)
$$

and a rapidity separation $\eta$ (with $e^{\eta} \ll 1$ ), the gluonic field can be split between "fast" (quantum part) and "slow" (classical part) as illustrated in Fig. 1:

$$
\begin{array}{rlr}
\mathscr{A}^{\mu a}\left(k^{+}, k^{-}, \vec{k}\right) & =A_{\eta}^{\mu a}\left(\left|k^{+}\right|>e^{\eta} p^{+}, k^{-}, \vec{k}\right) & \text { quantum part } \\
& +b_{\eta}^{\mu a}\left(\left|k^{+}\right|<e^{\eta} p^{+}, k^{-}, \vec{k}\right) & \text { classical part. }
\end{array}
$$

In the boosted projectile frame, the classical part $b^{\mu}$ has a particularly simple Lorentz structure, as

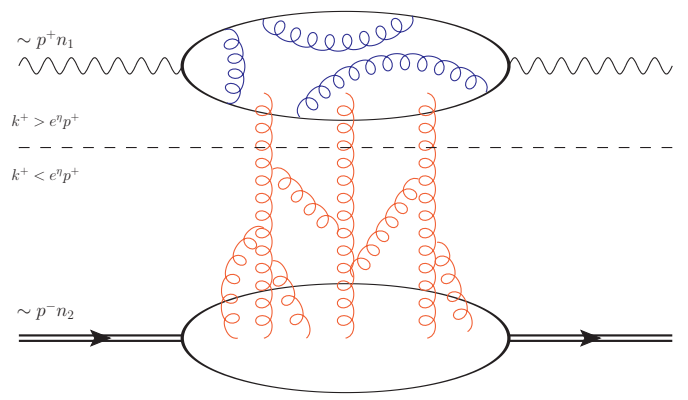

Figure 1: Splitting between quantum and classical parts.

illustrated in Fig. 2. Multiple interactions with the target can then be resummed into path-ordered Wilson lines $U_{\bar{z}_{i}}$ attached to each parton crossing lightcone time $x^{+}=0$ :

$$
U_{\vec{z}_{i}}^{\eta}=P e^{i g \int b_{\eta}^{-}\left(z_{i}^{+}, \vec{z}_{i}\right) d z_{i}^{+}}
$$

Finally, a factorized picture arises, which allows for a description of the scattering amplitude as a convolution, in transverse space, of matrix elements of the Wilson line operators acting on the 


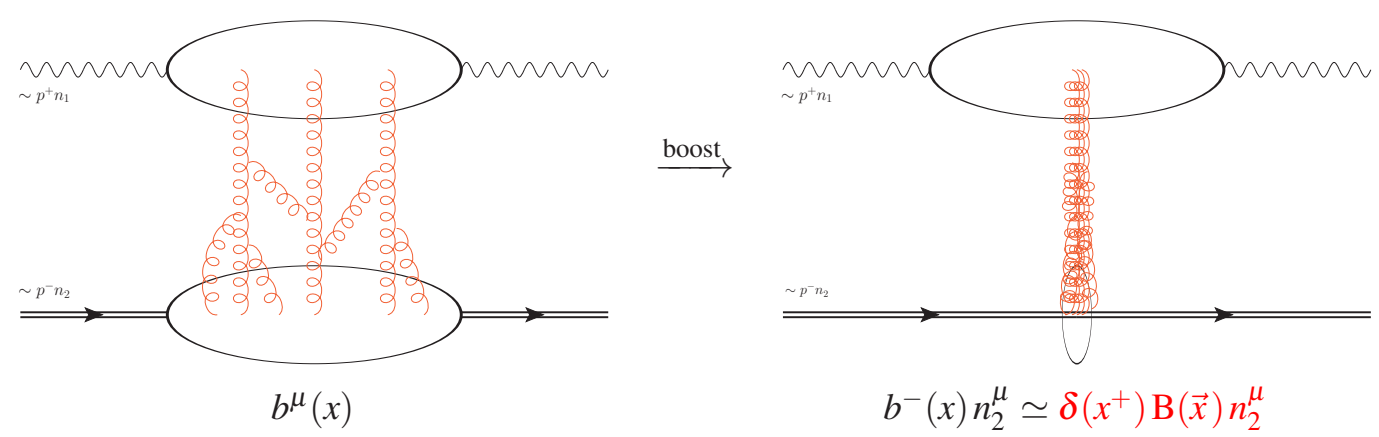

Figure 2: The shockwave approximation after a large longitudinal boost to the projectile frame.

target states with the impact factor describing the scattering of the projectile off the classical field:

$$
\mathscr{A}=\int d \vec{z}_{1} \ldots d \vec{z}_{n} \Phi\left(\vec{z}_{1}, \ldots, \vec{z}_{n}\right)\left\langle P^{\prime}\left|U_{\vec{z}_{1}} \ldots U_{\vec{z}_{n}}\right| P\right\rangle .
$$

The Wilson line operators evolve with $\eta$ through the Balitsky hierarchy [2], which includes non linear terms responsible for gluonic saturation. Equivalently to this high energy operator expansion, a functional approach has been developped, known as the color glass condensate formulation [3], governed by the JIMWLK evolution equation $[4,5,6,7,8,9,10,11,12]$.

Focusing on color-singlet exchange, exclusive diffraction allows one to probe the impact parameter $b_{\perp}$-dependence of the non-perturbative scattering amplitude. We went for the first time beyond leading-order (LO) in computing impact factors at next-to-leading order (NLO) in the case of diffractive exclusive dijet $[13,14]$ and light vector meson [15] production in arbitrary kinematics. A noticeable outcome is the fact that besides an intermediate color-dipole made by the $q \bar{q}$ pair, a configuration made of two dipoles is involved when an additional gluon at NLO goes through the shock-wave.

\section{Exclusive diffractive dijet electroproduction at HERA}

We investigated the ZEUS diffractive exclusive dijet measurements performed at HERA [16], see details in Ref. [17]. We denote as $W$ the $\gamma^{*} P$ total energy in the c.m.f., $Q^{2}$ the (opposite) $\gamma^{*}$ virtuality, and $M$ the mass of the diffractive dijet system. At LO, the $\gamma_{L}^{*} P$ cross-section

$$
\left.\frac{d \sigma_{0 L L}}{d t}\right|_{t=0}=\frac{1}{2(2 \pi)^{4}} \frac{4 \alpha Q_{q}^{2}}{N_{c}} \pi \int d x Q^{2} x^{2} \bar{x}^{2} \int d^{2} r K_{0}(\sqrt{x \bar{x}} Q r)^{2} F(\vec{r})^{2}
$$

is expressed through the forward dipole matrix element

$$
F\left(z_{\perp}\right)=\left.\frac{\left\langle P^{\prime}\left(p_{0}^{\prime}\right)\left|T\left(\operatorname{Tr}\left(U_{\frac{z_{\perp}}{2}} U_{-\frac{z_{\perp}}{2}}^{\dagger}\right)-N_{c}\right)\right| P\left(p_{0}\right)\right\rangle}{2 \pi \delta\left(p_{00^{\prime}}^{-}\right)}\right|_{p_{0} \rightarrow p_{0}^{\prime}}=N_{c} \sigma_{0}\left(1-e^{-\frac{z^{2}}{4 R_{0}^{2}}}\right),
$$

where we use the Golec-Biernat-Wüsthoff (GBW) parametrization [18] in the last equality, therefore including saturation for dipoles of transverse size larger than $R_{0}$. Here

$$
R_{0}=\frac{1}{Q_{0}}\left(\frac{x_{P}}{a_{0}}\right)^{\frac{\lambda}{2}}
$$


with

$$
x_{P}=\frac{Q^{2}+M^{2}-t}{Q^{2}+W^{2}},
$$

which describes the fraction of the incident momentum lost by the proton or carried by the Pomeron exchanged in $t$-channel ${ }^{1}$

At NLO, besides the LO contribution described by the emission of a $q \bar{q}$ pair from an initial virtual photon which goes through the classical gluonic field of the proton, one should further include configurations in which the dijet system can be made of three partons (real contributions) as well as of two partons with a one loop correction (virtual contributions). The precise way one attributes two and three partons to dijets or trijets configurations goes through a jet algorithm. ZEUS used the exclusive $k_{t}$-jet algorithm [20]. Let $E_{i}, E_{j}$, be the particle's energies and $\theta_{i j}$ the relative angle between them in the c.m.f, the distance between two particles is defined as

$$
d_{i j}=2 \min \left(E_{i}^{2}, E_{j}^{2}\right) \frac{1-\cos \theta_{i j}}{M^{2}}=\min \left(\frac{E_{i}}{E_{j}}, \frac{E_{j}}{E_{i}}\right) \frac{2 p_{i} \cdot p_{j}}{M^{2}} .
$$

The two particles then belong to one jet if $d_{i j}<y_{c u t}$, where $y_{c u t}$ regularizes both soft and collinear singularities. In practice, $y_{c u t}=0.15$ in ZEUS analysis, and we rely on a small $y_{\text {cut }}$ approximation.

The cuts used by ZEUS are $5 \mathrm{GeV}<Q, 5 \mathrm{GeV}<M_{2 j e t s}<25 \mathrm{GeV}, 2 \mathrm{GeV}<p_{\perp \text { min }}$. At Born level, this removes the aligned jets configurations $x \lesssim \frac{1}{\max \left(Q^{2}, M^{2}\right) R_{0}^{2}} \ll 1$, the leading twist contribution which normally dominates in the GBW saturation model. Besides, the typical hard scale in the impact factor is larger than $p_{\perp \text { min }}^{2}>Q_{s}^{2}$, justifying an expansion in powers of $Q_{s}$ : ZEUS experiment is dominated by the linear BFKL regime. We restrict ourselves to the dominant contributions:

- Born cross section with soft and collinear corrections

- real correction with dipole $\times$ dipole, dipole $\times$ double dipole, and double dipole $\times$ double dipole contributions for the gluon dipole dijet configuration.

The sum of these contributions is compared with ZEUS data for cross-section in Fig. 3, as a function of the Bjorken variable $\beta$ normalized to the pomeron momentum

$$
\beta=\frac{Q^{2}}{Q^{2}+M^{2}-t} \simeq \frac{Q^{2}}{Q^{2}+M^{2}} \quad \text { at small } t .
$$

One gets a good agreement with data at large $\beta$, while at small $\beta$ there is a poor agreement with data, as for the two gluon model of Ref. [21]. We get similar conclusions for the azimuthal distribution of the jets. This calls for an inclusion of the remaining nonenhanced contributions (the nonsingular part of the virtual corrections, and the remaining part of the real one).

\section{Conclusion}

We provided the first full NLO computation of the $\gamma^{(*)} \rightarrow$ jet jet and $\gamma_{L, T}^{(*)} \rightarrow \rho_{L}$ impact factors. This can be adapted for twist $3 \gamma_{L, T}^{(*)} \rightarrow \rho_{T}$ NLO production in the Wandzura-Wilczek approximation, removing factorization breaking end-point singularities even at NLO for a process which

\footnotetext{
${ }^{1}$ A detailed analysis using various models including saturation has been recently performed at LO in Ref. [19].
} 


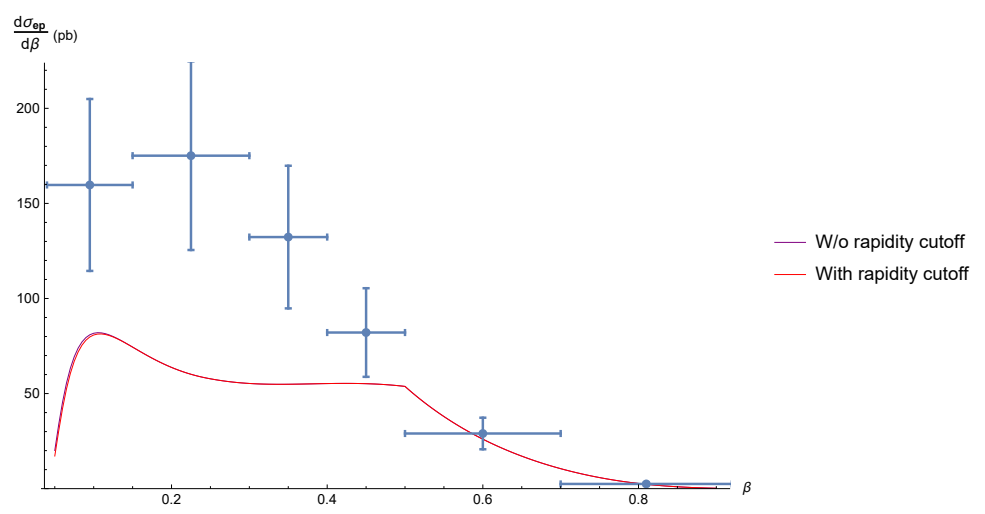

Figure 3: Born and total gluon dipole contributions to cross section.

would not factorize in a full collinear factorization scheme [22, 23]. For dijet electroproduction, in the small $y_{\text {cut }}$ limit of the exclusive $k_{t}$-jet algorithm, and for large $\beta$, a good agreement between the GBW model (in the small $Q_{s}$ expansion) combined with our NLO impact factor and ZEUS data is obtained. This is a good sign that perturbative Regge-like descriptions are favored with respect to collinear type descriptions. Finally, one should note that within ZEUS kinematical cuts, the linear BFKL regime dominates, while EIC should give a direct access to the saturated region.

\section{Acknowledgments}

This project has received funding from the European Union's Horizon 2020 research and innovation programme under grant agreement No 824093. The work of R. B. is supported by the U.S. Department of Energy, Office of Science, Office of Nuclear Physics, under contract No.DESC0012704, and in part by Laboratory Directed Research and Development (LDRD) funds from Brookhaven Science Associates. The work of L. S. is supported by the grant 2017/26/M/ST2/01074 of the National Science Center in Poland. He thanks CNRS, the LABEX P2IO, the GDR QCD. The project is also co-financed by the Polish National Agency for Academic Exchange. The work of A. V. G. is supported by the Russian Fund of Basic Research grant 19-02-00690 and the Ministry of Education and Science of Russia.

\section{References}

[1] J. C. Collins, Proof of factorization for diffractive hard scattering, Phys. Rev. D57 (1998) 3051-3056, [hep-ph/9709499].

[2] I. Balitsky, Operator expansion for high-energy scattering, Nucl. Phys. B463 (1996) 99-160, [hep-ph/9509348].

[3] L. D. McLerran and R. Venugopalan, Green's functions in the color field of a large nucleus, Phys. Rev. D50 (1994) 2225-2233, [hep-ph / 9402335$].$

[4] J. Jalilian-Marian, A. Kovner, A. Leonidov, and H. Weigert, The BFKL equation from the Wilson renormalization group, Nucl. Phys. B504 (1997) 415-431, [hep-ph/ 9701284 ]. 
[5] J. Jalilian-Marian, A. Kovner, A. Leonidov, and H. Weigert, The Wilson renormalization group for low x physics: Towards the high density regime, Phys. Rev. D59 (1999) 014014, [hep-ph/9706377].

[6] J. Jalilian-Marian, A. Kovner, and H. Weigert, The Wilson renormalization group for low x physics: Gluon evolution at finite parton density, Phys. Rev. D59 (1999) 014015, [hep-ph/970 9432 ].

[7] J. Jalilian-Marian, A. Kovner, A. Leonidov, and H. Weigert, Unitarization of gluon distribution in the doubly logarithmic regime at high density, Phys. Rev. D59 (1999) 034007, [hep-ph/980 462 ].

[8] A. Kovner, J. G. Milhano, and H. Weigert, Relating different approaches to nonlinear QCD evolution at finite gluon density, Phys. Rev. D62 (2000) 114005, [hep-ph/ 0004014 ].

[9] H. Weigert, Unitarity at small Bjorken x, Nucl. Phys. A703 (2002) 823-860, [hep-ph/ 0004044 ].

[10] E. Iancu, A. Leonidov, and L. D. McLerran, Nonlinear gluon evolution in the color glass condensate. I, Nucl. Phys. A692 (2001) 583-645, [hep-ph/ 0011241$].$

[11] E. Iancu, A. Leonidov, and L. D. McLerran, The renormalization group equation for the color glass condensate, Phys. Lett. B510 (2001) 133-144, [hep-ph/ 0102009 ].

[12] E. Ferreiro, E. Iancu, A. Leonidov, and L. McLerran, Nonlinear gluon evolution in the color glass condensate. II, Nucl. Phys. A703 (2002) 489-538, [hep-ph/0109115].

[13] R. Boussarie, A. Grabovsky, L. Szymanowski, and S. Wallon, Impact factor for high-energy two and three jets diffractive production, JHEP 1409 (2014) 026, [arXiv : 1405 . 7676].

[14] R. Boussarie, A. V. Grabovsky, L. Szymanowski, and S. Wallon, On the one loop $\gamma^{(*)} \rightarrow q \bar{q}$ impact factor and the exclusive diffractive cross sections for the production of two or three jets, JHEP 11 (2016) 149, [arXiv: 1606.0041$].$

[15] R. Boussarie, A. V. Grabovsky, D. Yu. Ivanov, L. Szymanowski, and S. Wallon, Next-to-Leading Order Computation of Exclusive Diffractive Light Vector Meson Production in a Saturation Framework, Phys. Rev. Lett. 119 (2017), no. 7 072002, [arXiv: 1612.0802 ].

[16] ZEUS Collaboration, H. Abramowicz et. al., Production of exclusive dijets in diffractive deep inelastic scattering at HERA, Eur. Phys. J. C76 (2016), no. 1 16, [arXiv: 1505 . 0578].

[17] R. Boussarie, A. V. Grabovsky, L. Szymanowski, and S. Wallon, Towards a complete next-to-logarithmic description of forward exclusive diffractive dijet electroproduction at HERA: real corrections, Phys. Rev. D100 (2019), no. 7 074020, [arXiv: 1905 . 0737].

[18] K. Golec-Biernat and M. Wüsthoff, Saturation effects in deep inelastic scattering at low $Q^{2}$ and its implications on diffraction, Phys. Rev. D59 (1999) 014017, [hep-ph / 9807513 ].

[19] F. Salazar and B. Schenke, Diffractive dijet production in impact parameter dependent saturation models, Phys. Rev. D100 (2019), no. 3 034007, [arXiv: 1905.0376 ].

[20] N. Brown and W. J. Stirling, Finding jets and summing soft gluons: A New algorithm, Z. Phys. $\mathbf{C 5 3}$ (1992) 629-636.

[21] J. Bartels, J. R. Ellis, H. Kowalski, and M. Wüsthoff, An analysis of diffraction in deep-inelastic scattering, Eur. Phys. J. C7 (1999) 443-458, [hep-ph/98034 97].

[22] I. V. Anikin, D. Yu. Ivanov, B. Pire, L. Szymanowski, and S. Wallon, On the description of exclusive processes beyond the leading twist approximation, Phys. Lett. B682 (2010) 413-418, [arXiv:0903.4797].

[23] I. V. Anikin, D. Yu. Ivanov, B. Pire, L. Szymanowski, and S. Wallon, QCD factorization of exclusive processes beyond leading twist: $\gamma_{T}^{*} \rightarrow \rho_{T}$ impact factor with twist three accuracy, Nucl. Phys. $\mathbf{B 8 2 8}$ (2010) 1-68, [arXiv:0909.4090]. 